\title{
Simple Method to Change the Magnetic Resonant Frequencies of Short Wire Pairs
}

\section{Yoshihiro Kokubo}

Graduate School of Engineering, University of Hyogo, Himeji-shi, Japan.

Email: kokubo@eng.u-hyogo.ac.jp

Received February 23 ${ }^{\text {rd }}, 2013$; revised March 24 ${ }^{\text {th }}$, 2013; accepted April $8^{\text {th }}$, 2013

Copyright (c) 2013 Yoshihiro Kokubo. This is an open access article distributed under the Creative Commons Attribution License, which permits unrestricted use, distribution, and reproduction in any medium, provided the original work is properly cited.

\begin{abstract}
Short wire pairs are simple metamaterial structures. This structure includes a dielectric substrate with metal strips on both sides, of which the electric and magnetic resonant frequencies can be controlled by adjusting the length of the metallic wires. However, to vary the magnetic resonant frequency requires a change in the length of the strip and another patterned photomask. In this investigation, a simple method is introduced that requires only one patterned photomask by shifting the position of faced wire pairs up and down.
\end{abstract}

Keywords: Metallic Waveguide; Metamaterial; Short Wire Pairs

\section{Introduction}

A left-handed material (LHM) was demonstrated by D. R. Smith et al. [1] that consisted of an array of conducting, nonmagnetic elements for achieving a negative effective permeability $\mu_{r}$, and an array of conducting continuous wires for achieving a negative effective permittivity $\varepsilon_{r}$, the simultaneous combination of which had never before been observed in any previously known material.

One type of metamaterials, referred to as short wire pairs, has a very simple structure [2]. This structure has a dielectric substrate with different metal strips on both sides, of which the electric and magnetic resonant frequencies can be controlled by adjusting the length of each metallic wire. A negative effective $\varepsilon_{r}$ and $\mu_{r}$ were observed at the same frequency of $14 \mathrm{GHz}$, leading to a negative refractive index. However, to vary the magnetic resonant frequency requires a change in the length of the metal strip and thus another patterned photomask.

We propose a simpler method and structure that requires only one pattern photomask, even if the magnetic resonant frequency is to be changed.

\section{Structure of Short Wire Pairs}

To evaluate the characteristics of short wire pairs, the structures were inserted into a metallic wave guide parallel to each other and the $S$ parameters were measured. The effective $\mu_{r}$ can be calculated using the $S$ parameters
[3]. Although this method is not accurate at the magnitude of the effective $\varepsilon_{r}$ and $\mu_{r}$, the resonant frequencies are accurate and can be easily calculated.

After obtaining $S_{11}$ and $S_{21}$ under conditions of $n \cdot k_{0} \cdot d \ll 1$, the complex effective $\varepsilon_{r}$ and $\mu_{r}$ are given by:

$$
\begin{aligned}
& \varepsilon_{r} \approx \frac{2}{j k_{0} d} \frac{1-\left(S_{21}+S_{11}\right)}{1+\left(S_{21}+S_{11}\right)} \\
& \mu_{r} \approx \frac{2}{j k_{0} d} \frac{1-\left(S_{21}-S_{11}\right)}{1+\left(S_{21}-S_{11}\right)}
\end{aligned}
$$

where $k_{0}=\omega / c, n=\sqrt{\varepsilon_{r}} \sqrt{\mu_{r}}$, and $d, \omega$, and $c$ represent the thickness of the metamaterial unit cell, the angular frequency and the velocity of light in free space, respectively.

The details of the metamaterial used for the calculation model are shown in Figure 1. Forty five short wire pairs were placed in a metallic wave guide (WRI-100 or WR-90: $22.9 \mathrm{~mm} \times 10.2 \mathrm{~mm}$ ) parallel to each other. The dielectric substrate used was an Arlon Diclad880 $\left(\varepsilon_{r}=2.17, \tan \delta=0.0009,0.508 \mathrm{~mm}\right.$ thick $)$ with thin copper films fixed on both sides. The Femtet software package [4], which employs the finite element method, was used for the calculations. The results of calculations for a wire length of $\ell=9.6 \mathrm{~mm}$ are shown in Figure 2. The magnetic resonant frequency is around $10.2 \mathrm{GHz}$. Complex permeability is represented by $\mu_{r}=\mu_{r}^{\prime}-j \cdot \mu_{r}^{\prime \prime}$, 


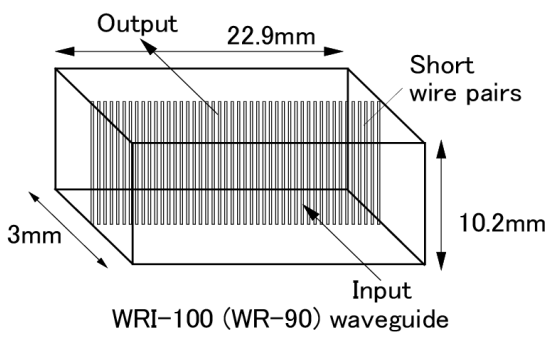

(a)
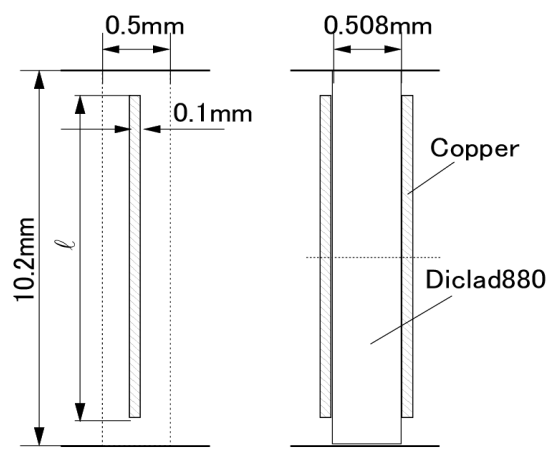

(b)

Figure 1. Profile of a metamaterial. (a) Schematic diagram of short wire pairs in a metallic waveguide; (b) Detailed profile of metal wires on the dielectric substrate.

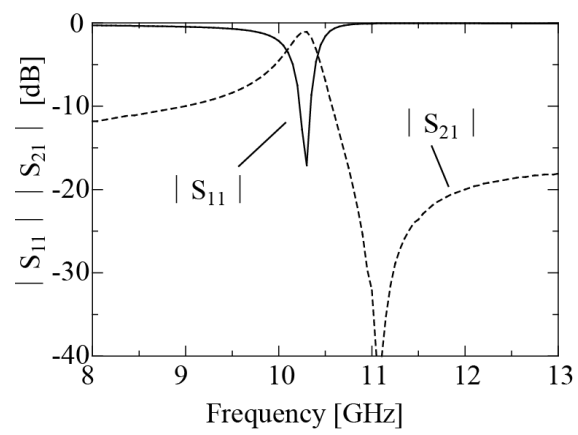

(a)

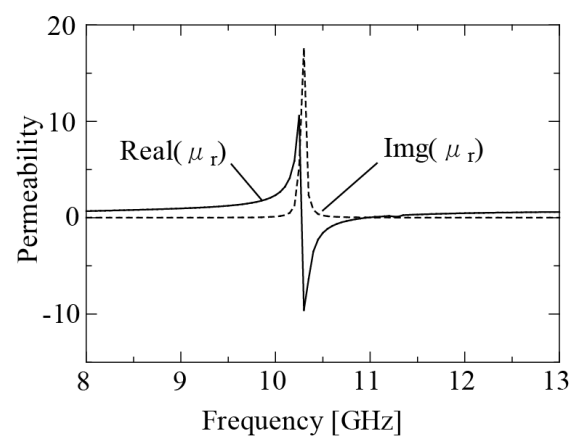

(b)

Figure 2. Calculation results for the (a) $S$ parameters; and (b) permeability of short wire pairs with $\ell=9.6 \mathrm{~mm}$.

where $\mu_{r}^{\prime}$ is the real part of complex permeability and $\mu_{r}^{\prime \prime}$ is the imaginary part. $\mu_{r}^{\prime}$ is changed significantly from a positive value to a negative value around the resonant frequency. On the other hand, $\mu_{r}^{\prime \prime}$ peaks at the resonant frequency and is very small at frequencies distant from the resonant frequency.

The $S$ parameters of the short wire pair metamaterial in a metallic waveguide connected to a HP-8510B network analyzer were measured using a coaxial-waveguide converter. The metamaterial sample is shown in Figure 3. The experimental results shown in Figure $\mathbf{4}$ are almost the same as those shown in Figure 2, which confirmed the reliability of the calculation.

\section{Simple Method to Change the Magnetic Resonant Frequencies}

The length of the short wire pairs $\ell$, was changed to 7

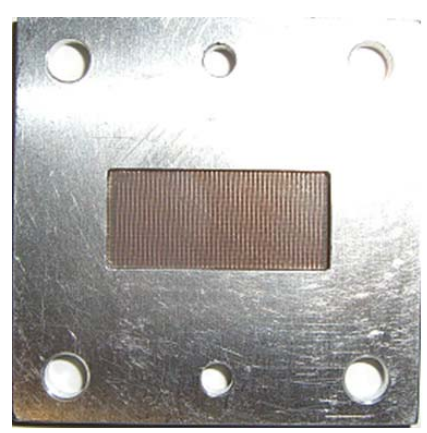

(a)

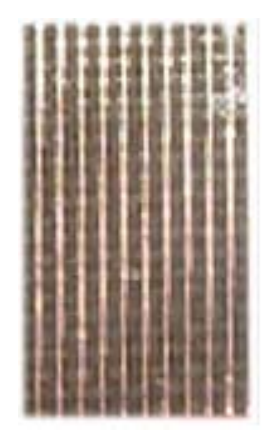

(b)
Figure 3. Sample of forty five short wire pairs as a metamaterial: (a) in a frame; and (b) magnified image.

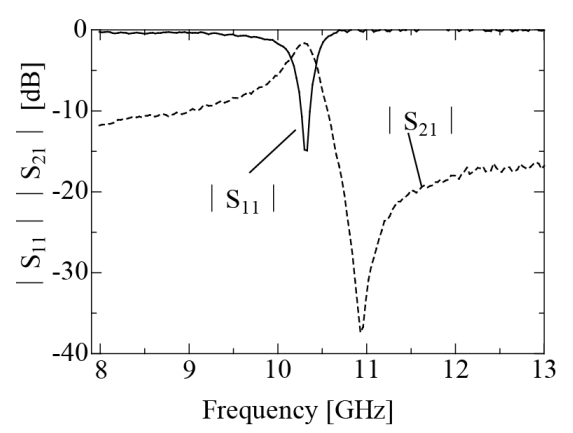

(a)

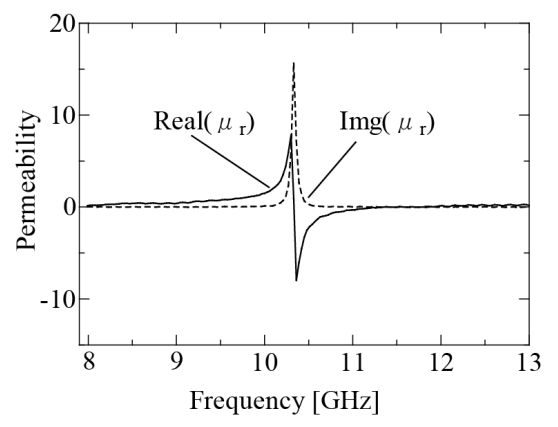

(b)

Figure 4. Experimental results for the (a) $S$ parameters, and (b) permeability of short wire pairs. 
$\mathrm{mm}$ in Figure 1 to change the magnetic resonant frequency. The results calculated for the $S$ parameters and permeability using the Femtet package are shown in Figure 5. The magnetic resonant frequency is around 13.8 $\mathrm{GHz}$, which is higher than that in Figure 2. To increase the magnetic resonant frequencies without changing the wire length of $\ell=7 \mathrm{~mm}$, the wire pairs facing each other are shifted up and down, as shown in Figure 6, where $\Delta$ represents the distance between the centers of the faced wire pairs. Figure 7 shows the $S$ parameters and permeability of short wire pairs with $\Delta=1.0 \mathrm{~mm}$. The magnetic resonant frequency is around $16.7 \mathrm{GHz}$, which is higher than that in Figure 5.

Figure 8 shows magnetic resonant frequencies with varying $\Delta$ and constant $\ell(=7 \mathrm{~mm})$ calculated using

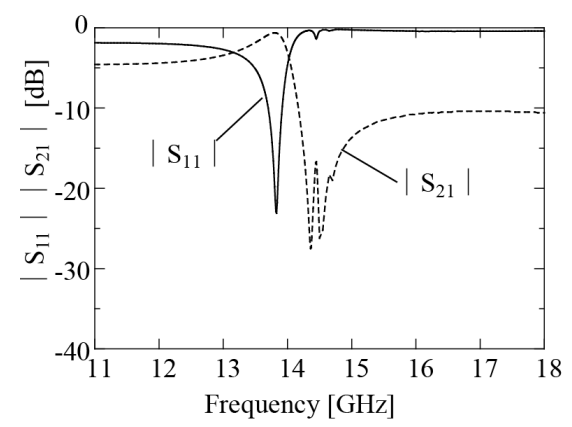

(a)

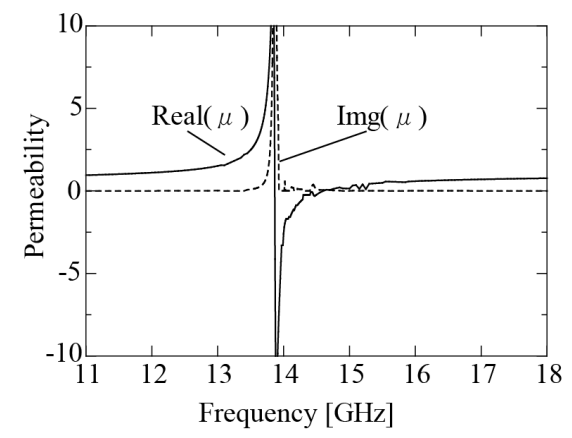

(b)

Figure 5. Calculation results for the (a) $S$ parameters; and (b) permeability of short wire pairs with $\ell=7 \mathrm{~mm}$.

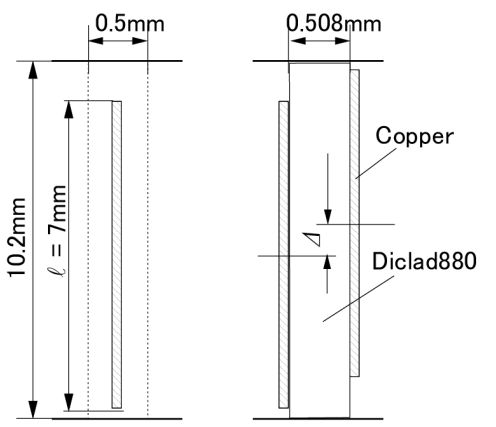

Figure 6. Profiles for the offset $\Delta$, between the centers of faced wire pairs.

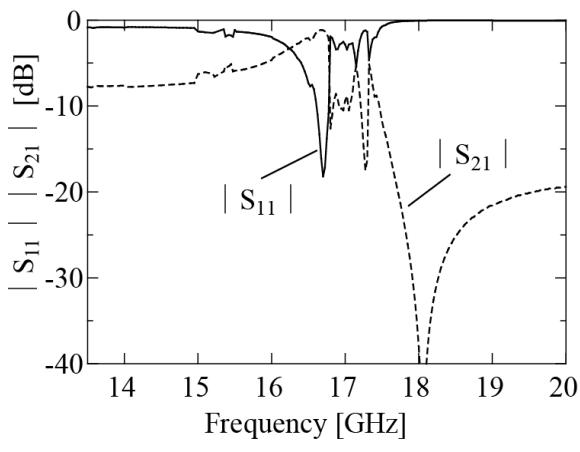

(a)

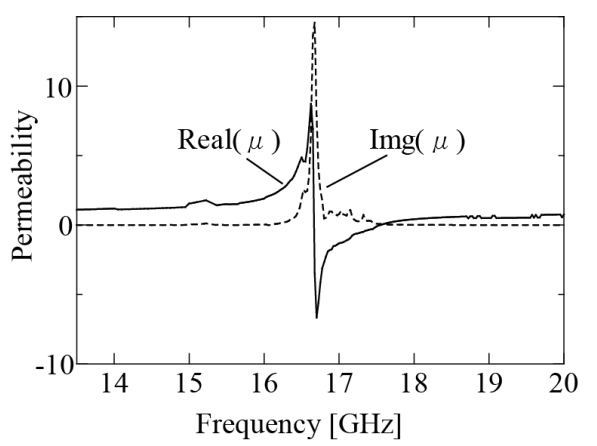

(b)

Figure 7. Calculation results for the (a) $S$ parameters, and (b) permeability of short wire pairs with $\ell=7 \mathrm{~mm}$ and $\Delta=1.0 \mathrm{~mm}$.

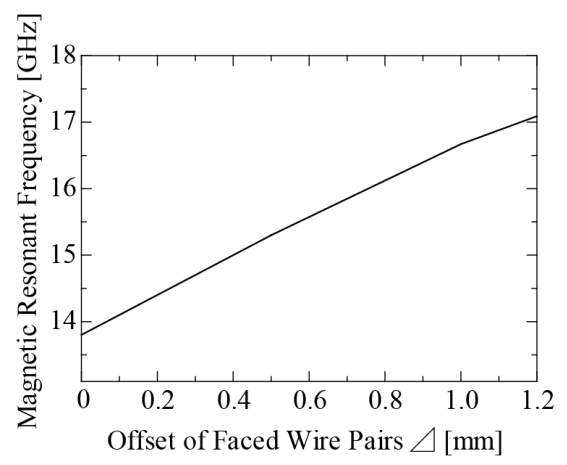

Figure 8. Magnetic resonant frequencies versus offset of faced wire pairs, $\Delta$.

Femtet. In this way, the magnetic resonant frequency can be changed without changing $\ell$, but by changing $\Delta$.

\section{Conclusion}

The magnetic resonant frequency of a short wire pair metamaterial could be changed by shifting the position of short wire pairs facing each other. Another patterned photomask is generally required to change the magnetic resonant frequency of a short wire pair metamaterial. However, this is a simple method that requires only one patterned photomask and further design for the positioning of short wire pairs in relation to each other. 


\section{Acknowledgements}

This work was supported by KAKENHI (22560336).

\section{REFERENCES}

[1] D. R. Smith, W. J. Padilla, D. C. Vier, S. C. Nemat-Nasser and S. Schultz, "Composite Medium with Simultaneously Negative Permeability and Permittivity,” Physical Review Letters, Vol. 84, No. 18, 2000, pp. 4184-4187. doi:10.1103/PhysRevLett.84.4184
[2] J. Zhou, L. Zhang, G. Tuttle, T. Koschny and C. M. Soukoulis, "Negative Index Materials Using Simple Short Wire Pairs,” Physical Review B, Vol. 73, 2006, pp. 1-4, Article ID: 041101, doi:10.1103/PhysRevB.73.041101

[3] R. W. Ziolkowski, "Design, Fabrication, and Testing of Double Negative Metamaterials," IEEE Tranaction on. Antennas and Propagation, Vol. 51, No. 7, 2003, pp. 1516-1529. doi:10.1109/TAP.2003.813622

[4] Murata Software Co., Ltd., "The Finite Element Analysis Software Package Femtet ${ }^{\circledR}$ Ver.11.1.1,” 2012. 